\title{
Observation of Continuous and Non-continuous Laser Induced Periodic Structures on Silicon
}

\author{
Pravakar Satapathy*, Rudrashish Panda*, Rajashree Sahoo*, Mukesh Kumar Shukla**, Lizina Khatua***, Pratap Kumar Sa- \\ hoo**, Ritwick Das $^{* *}$ and Susanta Kumar Das* \\ * Dept. of Physics, School of Applied Sciences, KIIT Deemed to be University, Odisha, 751024 \\ ** School of Physical Sciences, NISER, Bhubaneswar, HBNI, Odisha, 752050, India \\ ${ }^{* * *}$ School of Electronics Engineering, KIIT University, Odisha, 751024, India \\ E-mail: skdasfpy@kiit.ac.in
}

In this work we have demonstrated the formation of continuous and non-continuous laserinduced periodic structures on silicon substrate (SiLIPS) using nanosecond (ns) laser. The continuous SiLIPS were generated in air by linearly polarized $\sim 2$ ns laser pulses. The optimized fluence, repetition rate and scanning velocity for generation of such structures are $0.4 \mathrm{~J} / \mathrm{cm}^{2}, 40 \mathrm{kHz}$ and 0.5 $\mathrm{mm} / \mathrm{s}$ respectively. The period of the SiLIPS $(900 \mathrm{~nm})$ was found to be close to the laser wavelength and the orientation was perpendicular to the polarization of the laser beam. Further, in this work we have also shown the appearance of non-continuous SiLIPS in localized region. Based on this observation, discussion is given on the mechanism of SiLIPS formation.

DOI: $10.2961 / \mathrm{j} \operatorname{lmn} .2018 .03 .0001$

Keywords: Nanosecond laser, laser induced black Si, Laser induced periodic structure, Mechanism of ripple formation, Large area laser structuring

\section{Introduction}

The laser induced periodic structures (LIPS) generation is a phenomenon by which periodic structures can appear instantaneously on a surface and volume of any kind of solid state material when laser pulses of appropriate energy and number is irradiated on it. This is a kind of top down approach to produce highly reproducible nano/microstructures without going for any sophisticated process of lithography. However, un-like the other top down method, this method is much simpler and cost effective. Depending on the involved physical mechanisms, the process of LIPS formation can be driven by feedback loops leading to self-organization processes. This is found to be a universal phenomenon and has been used for periodic structure generation at the surface of various materials like metals [1], semi-conductors [2-3], dielectrics [4], ceramics [5-6] and polymers [6-7] etc. By adjusting the laser parameters like wavelength, polarization, pulse duration, pulse number and fluence; the shape, size and orientation of the created structures can be controlled very precisely [8].

The LIPS on silicon (SiLIPS) have been found to be very promising for various applications like generation of black Si, Surface enhanced Raman spectroscopy (SERS), Super hydrophobic surfaces generation etc [9-11]. Generally SiLIPS can be generated by using femtosecond (fs), picosecond (ps) and nanosecond (ns) lasers. Out of these, ns lasers are least expensive. So, several efforts have been made to generate SiLIPS with ns lasers [12-13]. However, because of thermal effect in such lasers, generally it is not a trivial task to generate uniform or continuous SiLIPS all across the scanned region. This problem is particularly very dominant in high repetition rate nanosecond laser although such laser has the advantage of facilitating the high speed processing. To avail this advantage, proper optimization particularly with respect to the repetition rate of laser pulses is required. In this work, we, therefore did the investigation on this regard and demonstrated that two types viz. continuous and non-continuous SiLIPS can be observed. We discussed the significance of such structures in terms of their potential applications and mechanism of formation.

\section{Experimental}

The schematic diagram of the experimental set up is shown in the Fig.1.

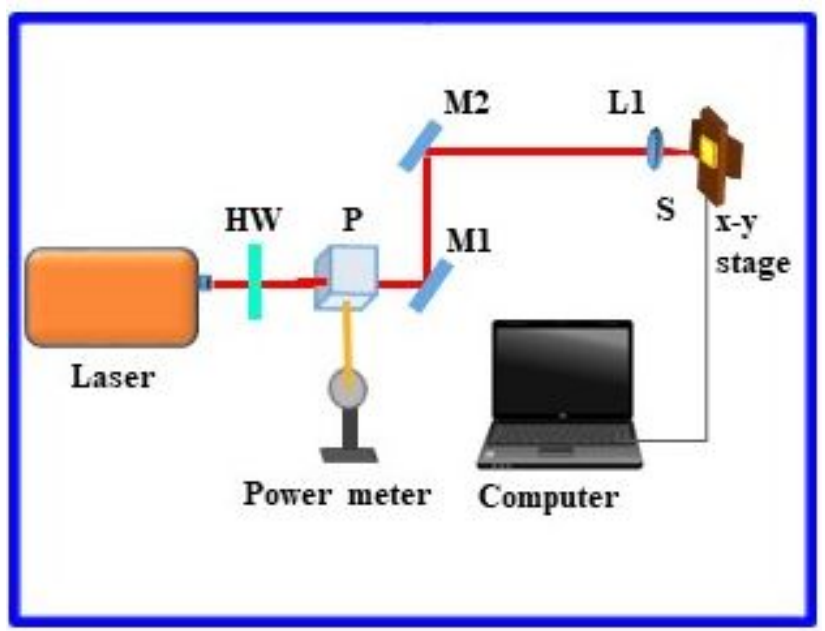

Fig.1 Fig.1 Schematic of experimental setup (HW = Half Wave plate, $\mathrm{P}=$ =Polarizer, M1,M2= Mirrors, L1= Focusing lens, $\mathrm{S}=$ Sample). 
A linearly polarized ns laser of wavelength $1064 \mathrm{~nm}$ was used for this work. The duration of the laser pulses is $\sim 2 \mathrm{~ns}$ and the repetition rate can be varied from $20 \mathrm{kHz}$ to 80 $\mathrm{kHz}$. The laser beam was stirred through two mirrors (M1, M2) and ultimately focused on to the sample (S) by a plano-convex lens of focal length $35 \mathrm{~mm}$ (L1). The sample was placed at the focal plane of the lens and the spot size (waist, $\omega_{0}$ ) of laser beam on its surface was $50 \mu \mathrm{m}$. For the linear processing, the sample was placed on a motorized $\mathrm{x}$ y stage (X-XY-LSM025A-KX13A-SQ3, Zaber technologies Inc.) which is controlled through an indigenously developed LabVIEW software program. Generally, a very precise control of fluence of laser is required to generate SiLIPS. Here laser fluence was controlled by a half wave plate $(\mathrm{HW})$ and a polarizer $(\mathrm{P})$. The beam reflected by po- larizer was measured continuously by a power meter. The whole experiment was carried out in air. The generated SiLIPS were characterized by The Field Emission Scanning Electron Microscope (FESEM). The outer part (20-40 $\mu \mathrm{m}$ off the centre) of the line imprinted on the sample was registered for these SEM images. In order to find the period of the SiLIPS the two dimensional Fast Fourier Transform (2D-FFT) of these images were done.

\section{Results and discussion}

The FESEM images of the typical SiLIPS generated in the optimized working condition are shown in Fig. 2(a) (low magnification) and Fig 2(b) (high magnification).
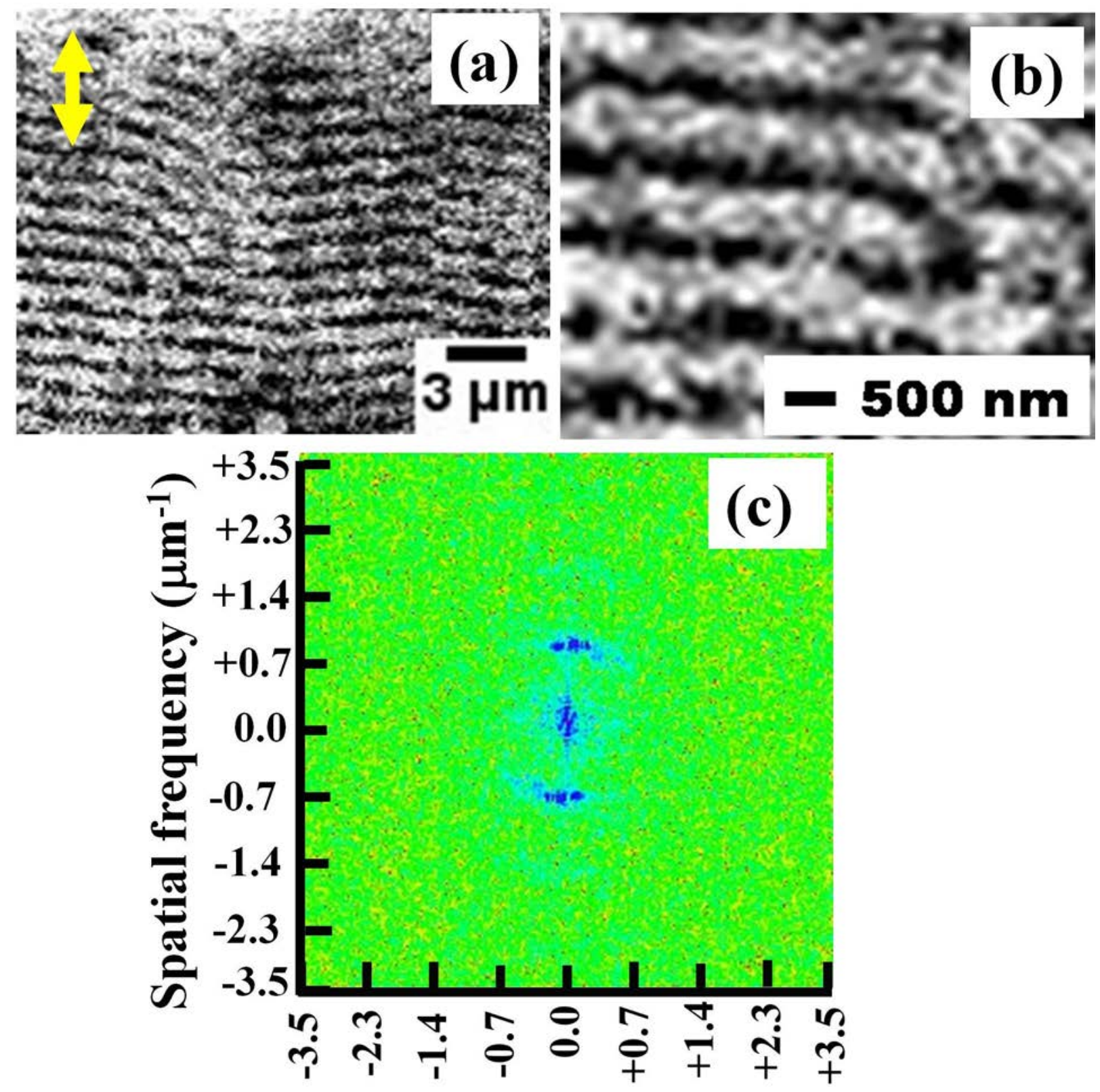

Spatial frequency $\left(\mu \mathrm{m}^{-1}\right)$

Fig. 2 (a)FESEM image of SiLIPS in low magnification (double sided arrow represent laser polarization), (b) FESEM images in high magnification, (c) 2D FFT of (a) 
In this optimized condition the repetition rate of the laser pulse was $40 \mathrm{kHz}$, the fluence and scanning velocity and number of overlapping pulses were $0.4 \mathrm{~J} / \mathrm{cm}^{2}, 0.5 \mathrm{~mm} / \mathrm{s}$ and 8000 respectively. The number of overlapping pulses were estimated by the formula $N=\frac{2 \omega_{0}}{v} R_{p}$ where, $\omega_{0}=$ beam spot size as mentioned above, $v=$ scanning velocity and $R_{p}=$ repetition rate of the laser pulses. As can be seen from Fig 2(a), the SiLIPS formed were uniform all across the processing line. The orientation of SiLIPS was found to be perpendicular to the polarization of the laser beam (shown as double sided arrow in Fig 2(a)). The 2D-FFT analysis of SiLIPS of Fig. 2(a) is shown in Fig. 2(c). From this 2DFFT analysis, the period is estimated to be around $900 \mathrm{~nm}$ which is close to the wavelength of the laser. This period of SiLIPS is found to be almost constant all over the area where they form.Nürnberger et al. and Huynh et al. [12-13] have also demonstrated the period to be close to wavelength using ns laser in SiLIPS. So, our result with high repetition rate is found to be well matched with those results.

It is important to note that there has been an effort on generation of SiLIPS using high repetition rate ns laser. However, in that work, the Si wafer was coated with the $\mathrm{SiO}_{2}$ layer [12]. On the other hand, we demonstrate highly uniform LIPS on bare Si surface in the present case. These highly uniform/continuous SiLIPS can found to be very useful for various applications like SERS, photovoltaic cell, creating hydrophobic Si surface and laser induced black Si etc.

Further, it is worthwhile note that we also performed the experiment with $80 \mathrm{kHz}$ and $20 \mathrm{kHz}$ repetition rate of the laser pulse. At $80 \mathrm{kHz}$, once again we observed the continuous SiLIPS (Fig 3(a)) across the entire scanned region of $\mathrm{Si}$. The fluence and scanning velocity in which these structures obtained were $0.32 \mathrm{~J} / \mathrm{cm}^{2}$ and $0.5 \mathrm{~mm} / \mathrm{s}$ respectively.
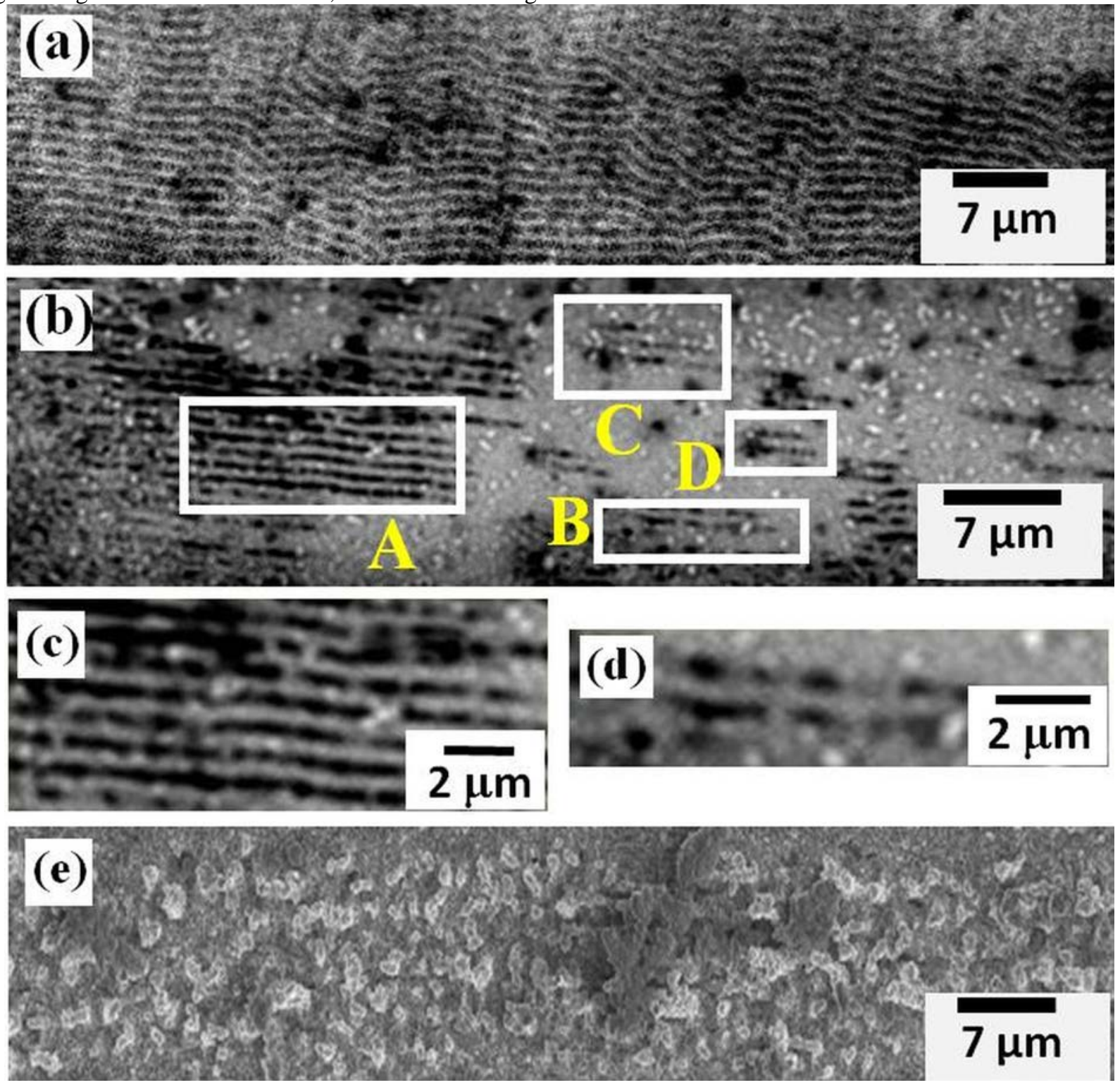

Fig.3 (a) FESEM image of continuous SiLIPS all across the processed line; (b) FESEM image of non-continuous SiLIPS, (c), (d) magnified images of region A and B of (b); (e) FESEM of molten region indicating non-appearance of SiLIPS 
The structures obtained for $20 \mathrm{kHz}$ are shown in Figure3(b). The fluence and scanning velocity and number of overlapping pulses in which these structures obtained were $0.4 \mathrm{~J} / \mathrm{cm}^{2}, 15 \mathrm{~mm} / \mathrm{s}$ and 134 respectively. As can be seen from the Fig. 3(b), the LIPS appeared only in some discrete regions like $\mathrm{A}, \mathrm{B}, \mathrm{C}$ and $\mathrm{D}$ etc. i.e. the LIPS are not continuous all across the processed region. The magnified images of region of A and B are shown in Fig. 3(c) and Fig. 3(d) respectively. From Fig 3(d) it revealed that in a given region also there discontinuity in the LIPS. These noncontinuous LIPS can generate only through the ablation of material in the localized region. Such localized or noncontinuous structures cannot be used for any application. However, appearance of such structures can give a direct indication on the mechanism of formation of SiLIPS. Particularly, it is of utmost importance to point out that, Nürnberger et al. have predicted melting of $\mathrm{Si}$ when excited with ns laser pulses and behaves like a metallic substance because of generation of high carrier density [12]. This metallic surface along with the adjacent dielectric layer support Surface Plasmon Polaritons (SPPs).These SPP modes couple to the incoming radiation giving rise to SiLIPS of period close to the wavelength of laser. The appearance of localized non-continuous SiLIPS, in our case, strongly contradicts this as it is formed without melting of Si which is substantiated from the image in Fig. 3(b)-3(d). The experiment was also done at $30 \%$ higher than the aforementioned optimized fluence for the repetition rate of $40 \mathrm{KHz}$. In this condition only melting regions were observed (Fig. 3(e)). This observation once again confirm the fact that melting is not essential for the LIPS formation. In other words we can conclude that upon the excitation of ns laser pulses the $\mathrm{Si}$ can behave like metallic surface without even going for melting process and can support SPP for generation of SiLIPS through localized ablation of materials like the case that has been reported for fs laser [14].

\section{Conclusions}

To conclude, in this work we have demonstrated the formation of continuous and non-continuous laser-induced periodic structures on silicon substrate using the ns laser. The uniform/continuous SiLIPS can found to be very useful for various applications like SERS, photovoltaic cell, creating hydrophobic Si surface and laser induced black Si etc. Further, in this work we have also shown the appearance of localized and non-continuous SiLIPS. This observation indicates that Si surface could exhibit metallic characteristics and consequently, could support SPP modes for generation of SiLIPS when excited by ns laser pulses even without going for melting.

\section{Acknowledgments}

SKD thanks Science \& Engineering Research Board (SERB), Govt. of India (Project File Number: EMR/2015/001175) for the financial support.

\section{References}

1. A.Y. Vorobyev and C. Guo:Appl. Phys. A, 86, (2007) 321.

2. M. Shen, J. E. Carey, C. H. Crouch, M. Kandyla, H. A. Stone, and E. Mazur:Nano Lett., 8, (2008) 2087.

3. B.Kumar and R. K. Soni:J. Phys. D: Appl. Phys., 41, (2008) 155303.

4. R.Taylor, C. Hnatovsky, and E. Simova:Laser \& Photon. Rev., 2, (2008) 26.

5. J. Bonse, H. Sturm, H. D. Schmidt, and W. Kautek:Appl. Phys. A, 71, (2000) 657.

6. H. Hiraoka, W. Y. Y. Wong, T. M. Wong, C. T. Hung, W. C. Loh, and F. M. Lee:J. Photopolymer Sci. Technol., 10, (1997) 205.

7. S. Baudach, J. Bonse, and W. Kautek:Appl. Phys. A, 69, (1999) S395.

8. K.W. Kolasinski:Curr. Opin. Solid State Mater. Sci., 11, (2007) 76.

9. R.Torres, T. E. Itina, V. Vervisch, M. Halbwax, T. Derrien, T. Sarnet, M. Sentis, J. Ferreira, F. Torregrosa, and L. Roux:AIP Conf. Proc., 1278, (2010) 576.

10. C.H. Lin, L. Jiang, H. Xiao, S. J. Chen, and H. L. Tsai:Opt. Lett., 35(17), (2010) 2937.

11. T.Baldacchini, J. E. Carey, M. Zhou, and E. Mazur:Langmuir, 22(11), (2006) 4917.

12. P.Nürnberger, H. M. Reinhardt, H. C. Kim, E. Pfeifer, M. Kroll, S. Müller, F. Yang, and N. A. Hampp:Appl. Surf. Sci., 425, (2017) 682.

13. T T D Huynh and N Semmar:Surf. Topogr.: Metrol. Prop., 5(3), (2017) 1.

14. J. Bonse, A. Rosenfeld, and J. Krüger: J. Appl. Phys., 106, (2009) 104910.

(Received: May 1, 2018, Accepted: September 9, 2018) 